\title{
On a Type of Para-Kenmotsu Manifold
}

\author{
T. Satyanarayana \\ Department of Mathematics \\ Pragati Engineering College, Surampalem, Near Peddapuram \\ Andhra Pradesh, India \\ tsn9talluri@gmail.com

\section{K. L. Sai Prasad*} \\ Department of Mathematics \\ GVP College of Engineering For Women, Visakhapatnam \\ Andhra Pradesh, India \\ klsprasad@yahoo.com
}

Copyright (C) 2013 T. Satyanarayana and K. L. Sai Prasad. This is an open access article distributed under the Creative Commons Attribution License, which permits unrestricted use, distribution, and reproduction in any medium, provided the original work is properly cited.

\begin{abstract}
The object of this paper is to study a class of almost para-contact metric manifold namely para-Kenmotsu (briefly p-Kenmotsu) manifold in which $\mathrm{R}(\mathrm{X}, \mathrm{Y}) . \mathrm{C}=0$ where $\mathrm{C}$ is the conformal curvature tensor of the manifold and $\mathrm{R}$ is the Riemannian curvature and $\mathrm{R}(\mathrm{X}$, $\mathrm{Y}$ ) is considered as a derivation of the tensor algebra at each point of the manifold for tangent vectors $\mathrm{X}$ and $\mathrm{Y}$.
\end{abstract}

\section{Mathematics Subject Classification: 53C15}

Keywords: Kenmotsu Manifold, Curvature Tensor, Ricci Tensor, Tangent Vector

\section{Introduction}

Sato [1] defined the notions of an almost para-contact Riemannian manifold. After that, T. Adati and K. Matsumoto [2] defined and studied para-Sasakian and SP-Sasakian manifolds which are regarded as a special kind of an almost contact Riemannian manifolds. Before Sato, Kenmotsu [3] defined a class of almost contact Riemannian manifolds. In 1995, Sinha and Sai Prasad [4] have defined a class of almost para-contact metric manifolds namely para-Kenmotsu (briefly p-Kenmotsu) and SP-Kenmotsu manifolds. They also have studied the curvature properties of $\mathrm{p}$-Kenmotsu manifold and the curvature properties of semisymmetric metric connection of SP-Kenmotsu manifold. 
Let $\mathrm{M}_{\mathrm{n}}$ be an $\mathrm{n}$-dimensional differentiable manifold equipped with structure tensors $(\phi, \xi, \eta)$ where $\phi$ is a tensor of type $(1,1), \xi$ is a vector field, $\eta$ is a 1 -form such that

$$
\begin{aligned}
\eta(\xi) & =1 \\
\phi^{2}(X) & =X-\eta(X) \xi ; \quad \bar{X}=\phi X
\end{aligned}
$$

Then $\mathrm{M}_{\mathrm{n}}$ is called an almost para-contact manifold.

Let ' $\mathrm{g}$ ' be the Riemannian metric satisfying such that, for all vector fields $\mathrm{X}$ and $\mathrm{Y}$ on $\mathrm{M}$,

$$
\begin{aligned}
& \mathrm{g}(\mathrm{X}, \xi)=\eta(X) \\
& \phi \xi=0, \eta(\phi X)=0, \text { rank } \phi=\mathrm{n}-1 \\
& \mathrm{~g}(\phi X, \phi Y)=\mathrm{g}(\mathrm{X}, \mathrm{Y})-\eta(\mathrm{X}) \eta(\mathrm{Y}) .
\end{aligned}
$$

Then the manifold $\mathrm{M}_{\mathrm{n}}$ is said to admit [1] an almost para-contact Riemannian structure $(\phi, \xi, \eta, g)$.

A manifold of dimension ' $n$ ' with Riemannian metric ' $g$ ' admitting a tensor field ' $\phi$ ' of type $(1,1)$, a vector field ' $\xi$ ' and a 1-form ' $\eta$ ' satisfying (1.1), (1.3) along with

$$
\begin{aligned}
& \left(\nabla_{X} \eta\right) Y-\left(\nabla_{Y} \eta\right) X=0 \\
& \left(\nabla_{X} \nabla_{Y} \eta\right) Z=[-g(X, Z)+\eta(X) \eta(Z)] \eta(Y)+[-g(X, Y)+\eta(X) \eta(Y)] \eta(Z) \\
& \nabla_{X} \xi \quad=\phi^{2} X=X-\eta(X) \xi
\end{aligned}
$$

is called a para-Kenmotsu manifold or briefly P-Kenmotsu manifold [4]. This paper deals with a type of $\mathrm{p}$-Kenmotsu manifold in which

$$
\mathrm{R}(\mathrm{X}, \mathrm{Y}) \cdot \mathrm{C}=0
$$

where $\mathrm{C}$ is the conformal curvature tensor of the manifold and $\mathrm{R}$ is the Riemannian curvature. Let $\left(\mathrm{M}_{\mathrm{n}}, \mathrm{g}\right)$ be an $\mathrm{n}$-dimensional Riemannian manifold admitting a tensor field ' $\phi$ ' of type $(1,1)$, a vector field ' $\xi$ ' and a 1-form ' $\eta$ ' satisfying

$$
\begin{aligned}
& \left(\nabla_{X} \eta\right) Y=g(X, Y)-\eta(X) \eta(Y) \\
& g(X, \xi)=\eta(X) \text { and }\left(\nabla_{X} \eta\right) Y=\varphi(\bar{X}, Y), \text { where } \varphi \text { is an associate of } \phi
\end{aligned}
$$

is called a special P-Kenmotsu manifold or briefly SP-Kenmotsu manifold [4]. In this paper it is proved that if in a P-Kenmotsu manifold $\left(M_{n}, g\right)(n>3)$ the relation (1.9) holds then the manifold is conformally flat and hence is an SP-Kenmotsu manifold. Also it is shown that a conformally symmetric P-Kenmotsu manifold $\left(\mathrm{M}_{\mathrm{n}}, \mathrm{g}\right)$ is an SP-Kenmotsu manifold for $\mathrm{n}>3$. (since it is known that $\mathrm{C}=0$ when $\mathrm{n}=3$, it has taken that $\mathrm{n}>3$ ). 
It is known that [4] in a P-Kenmotsu manifold the following relations hold:

$$
\begin{aligned}
& \mathrm{S}(\mathrm{X}, \xi)=-(\mathrm{n}-1) \eta(\mathrm{X}) \\
& \mathrm{g}[\mathrm{R}(\mathrm{X}, \mathrm{Y}) \mathrm{Z}, \xi]=\eta[\mathrm{R}(\mathrm{X}, \mathrm{Y}, \mathrm{Z})]=\mathrm{g}(\mathrm{X}, \mathrm{Z}) \eta(\mathrm{Y})-\mathrm{g}(\mathrm{Y}, \mathrm{Z}) \eta(\mathrm{X}) \\
& \mathrm{R}(\mathrm{X}, \xi)=-1 \\
& \mathrm{R}(\mathrm{X}, \xi, \xi)=-\mathrm{X}+\eta(\mathrm{X}) \xi \\
& \mathrm{R}(\mathrm{X}, \xi, \mathrm{X})=\xi \\
& \mathrm{R}(\xi, \mathrm{X}, \xi)=\mathrm{X} \\
& \mathrm{R}(\mathrm{X}, \mathrm{Y}, \xi)=\eta(\mathrm{X}) \mathrm{Y}-\eta(\mathrm{Y}) \mathrm{X} \text {; when } \mathrm{X} \text { is orthogonal to } \xi .
\end{aligned}
$$

where $\mathrm{S}$ is the Ricci tensor and $\mathrm{R}$ is the Riemannian curvature.

Moreover, it is also known that [4] a P-Kenmotsu manifold cannot be flat and a projectively flat P-Kenmotsu manifold i.e., a P-Kenmotsu manifold satisfying $\mathrm{R}(\mathrm{X}, \mathrm{Y}) \mathrm{W}=0$ is said to be Einstein manifold with the constant curvature $-n(n-1)$. The above results will be used in the next section.

\section{P-Kenmotsu manifold satisfying $R(X, Y) . C=0$}

We have

$$
\begin{aligned}
\mathrm{C}(\mathrm{X}, \mathrm{Y}) \mathrm{Z}=\mathrm{R}(\mathrm{X}, \mathrm{Y}) \mathrm{Z}-\frac{1}{\mathrm{n}-2}\left[\mathrm{~g}(\mathrm{Y}, \mathrm{Z}) \mathrm{QX}-\mathrm{g}(\mathrm{X}, \mathrm{Z}) \mathrm{QY}+\mathrm{S}\left(\mathrm{Y}_{y} \mathrm{Z}\right) \mathrm{X}-\mathrm{S}(\mathrm{X}, \mathrm{Z}) \mathrm{Y}\right]+ \\
\frac{\mathrm{r}}{(\mathrm{n}-1)(\mathrm{n}-2)}\left[\mathrm{g}\left(\mathrm{Y}_{y} \mathrm{Z}\right) \mathrm{X}-\mathrm{g}(\mathrm{X}, \mathrm{Z}) \mathrm{Y}\right]
\end{aligned}
$$

where ' $\mathrm{r}$ ' is the scalar curvature and ' $\mathrm{Q}$ ' is the symmetric endomorphism of the tangent space at each point corresponding to the Ricci tensor ' $S$ ' [5] i.e.,

$$
\mathrm{g}(\mathrm{QX}, \mathrm{Y})=\mathrm{S}(\mathrm{X}, \mathrm{Y}) \text {. }
$$

Then

$$
\begin{aligned}
& \eta(\mathrm{C}(\mathrm{X}, \mathrm{Y}) \mathrm{Z})=\mathrm{g}(\mathrm{C}(\mathrm{X}, \mathrm{Y}) \mathrm{Z}, \xi) \\
& =\frac{1}{\mathrm{n}-2}\left[\left(\frac{\mathrm{r}}{\mathrm{n}-1}+1\right)\left(\mathrm{g}\left(\mathrm{Y}_{,} \mathrm{Z}\right) \eta(\mathrm{X})-\mathrm{g}(\mathrm{X}, \mathrm{Z}) \eta(\mathrm{Y})\right)-\left(\mathrm{S}\left(\mathrm{Y}_{s} \mathrm{Z}\right) \eta(\mathrm{X})-\mathrm{S}(\mathrm{X}, \mathrm{Z}) \eta(\mathrm{Y})\right)\right] \text {. }
\end{aligned}
$$

Putting $Z=\xi$ in (2. 3), we get

$$
\eta(\mathrm{C}(\mathrm{X}, \mathrm{Y}) \xi)=0
$$


Again putting $X=\xi$ in (2.3), we get

$$
\eta(C(\xi, Y) Z)=\frac{1}{n-2}\left[\left(\frac{r}{n-1}+1\right) g\left(Y_{m} Z\right)-S(Y, Z)-\left(\frac{r}{n-1}+n\right) \eta(Y) \eta(Z)\right] \text {. }
$$

Now

$$
\begin{aligned}
(\mathrm{R}(\mathrm{X}, \mathrm{Y}) . \mathrm{C})(\mathrm{U}, \mathrm{V}) \mathrm{W} & =\mathrm{R}(\mathrm{X}, \mathrm{Y}) \mathrm{C}(\mathrm{U}, \mathrm{V}) \mathrm{W}-\mathrm{C}(\mathrm{R}(\mathrm{X}, \mathrm{Y}) \mathrm{U}, \mathrm{V}) \mathrm{W} \\
& -\mathrm{C}(\mathrm{U}, \mathrm{R}(\mathrm{X}, \mathrm{Y}) \mathrm{V}) \mathrm{W}-\mathrm{C}(\mathrm{U}, \mathrm{V}) \mathrm{R}(\mathrm{X}, \mathrm{Y}) \mathrm{W} .
\end{aligned}
$$

In virtue of (1.9) we get

$$
\begin{aligned}
\mathrm{R}(\mathrm{X}, \mathrm{Y}) \mathrm{C}(\mathrm{U}, \mathrm{V}) \mathrm{W}-\mathrm{C}(\mathrm{R}(\mathrm{X}, \mathrm{Y}) \mathrm{U}, \mathrm{V}) \mathrm{W} \\
-\mathrm{C}(\mathrm{U}, \mathrm{R}(\mathrm{X}, \mathrm{Y}) \mathrm{V}) \mathrm{W}-\mathrm{C}(\mathrm{U}, \mathrm{V}) \mathrm{R}(\mathrm{X}, \mathrm{Y}) \mathrm{W}=0 .
\end{aligned}
$$

Therefore

$$
\begin{aligned}
\mathrm{g}(\mathrm{R}(\xi, \mathrm{Y}) \mathrm{C}(\mathrm{U}, \mathrm{V}) \mathrm{W}, \xi)-\mathrm{g}(\mathrm{C}(\mathrm{R}(\xi, \mathrm{Y}) \mathrm{U}, \mathrm{V}) \mathrm{W}, \xi) \\
-\mathrm{g}(\mathrm{C}(\mathrm{U}, \mathrm{R}(\xi, \mathrm{Y}) \mathrm{V}) \mathrm{W}, \xi)-\mathrm{g}(\mathrm{C}(\mathrm{U}, \mathrm{V}) \mathrm{R}(\xi, \mathrm{Y}) \mathrm{W}, \xi)=0
\end{aligned}
$$

From this, it follows that

$$
\begin{aligned}
& \text { C }(\mathrm{U}, \mathrm{V}, \mathrm{W}, \mathrm{Y})-\eta(\mathrm{Y}) \eta(\mathrm{C}(\mathrm{U}, \mathrm{V}) \mathrm{W})+\eta(\mathrm{U}) \eta\left(\mathrm{C}\left(\mathrm{Y}_{s} \mathrm{~V}\right) \mathrm{W}\right)+\eta(\mathrm{V}) \eta(\mathrm{C}(\mathrm{U}, \mathrm{Y}) \mathrm{W}) \\
& +\eta(\mathrm{W}) \eta(\mathrm{C}(\mathrm{U}, V) \mathrm{Y})-g\left(Y_{s} U\right) \eta(\mathrm{C}(\xi, V) \mathrm{W})-g\left(Y_{s} V\right) \eta(\mathrm{C}(\mathrm{U}, \xi) \mathrm{W}) \\
& -g\left(Y_{s}, W\right) \eta(\mathrm{C}(\mathrm{U}, \mathrm{V}) \xi)=0
\end{aligned}
$$

where

$$
\text { 'C }(\mathrm{U}, \mathrm{V}, \mathrm{W}, \mathrm{Y})=\mathrm{g}(\mathrm{C}(\mathrm{U}, \mathrm{V}) \mathrm{W}, \mathrm{Y}) .
$$

Putting $\mathrm{Y}=\mathrm{U}$ in $(2.8)$ we get

$$
\begin{aligned}
& \mathrm{C}(\mathrm{U}, \mathrm{V}, \mathrm{W}, \mathrm{U})-\eta(\mathrm{U}) \eta(\mathrm{C}(\mathrm{U}, \mathrm{V}) \mathrm{W})+\eta(\mathrm{U}) \eta(\mathrm{C}(\mathrm{U}, \mathrm{V}) \mathrm{W})+\eta(\mathrm{V}) \eta(\mathrm{C}(\mathrm{U}, \mathrm{U}) \mathrm{W}) \\
& +\eta(\mathrm{W}) \eta(\mathrm{C}(\mathrm{U}, \mathrm{V}) \mathrm{U})-g\left(U_{s} U\right) \eta(\mathrm{C}(\xi, \mathrm{V}) \mathrm{W})-g\left(U_{s} V\right) \eta(\mathrm{C}(\mathrm{U}, \xi) W) \\
& -g\left(U_{s}, W\right) \eta(\mathrm{C}(\mathrm{U}, \mathrm{V}) \xi)=0 .
\end{aligned}
$$

Let $\left\{\mathrm{e}_{\mathrm{i}}\right\}, \mathrm{i}=1,2, \ldots, \mathrm{n}$ be an orthonormal basis of the tangent space at any point. Then the sum $1 \leq \mathrm{i} \leq \mathrm{n}$ of the relation (2.9) for $\mathrm{U}=\mathrm{e}_{\mathrm{i}}$ gives

$$
\eta(\mathrm{C}(\xi, v) \mathrm{W})=0 \text {. }
$$

By using (2.4), we have from (2. 8)

$$
\begin{aligned}
& \text { C }(\mathrm{U}, \mathrm{V}, \mathrm{W}, \mathrm{Y})-\eta(\mathrm{Y}) \eta(\mathrm{C}(\mathrm{U}, \mathrm{V}) \mathrm{W})+\eta(\mathrm{U}) \eta\left(\mathrm{C}\left(\mathrm{Y}_{s} \mathrm{~V}\right) \mathrm{W}\right)+\eta(\mathrm{V}) \eta\left(\mathrm{C}\left(\mathrm{U}_{s} \mathrm{Y}\right) \mathrm{W}\right) \\
& +\eta(\mathrm{W}) \eta(\mathrm{C}(\mathrm{U}, \mathrm{V}) \mathrm{Y})-g\left(Y_{s} U\right) \eta(\mathrm{C}(\xi, \mathrm{V}) \mathrm{W})-g\left(Y_{s} V\right) \eta\left(\mathrm{C}\left(\mathrm{U}_{s} \xi\right) \mathrm{W}\right)=0
\end{aligned}
$$

In virtue of (2.5) and (2.10) we have

$$
\mathrm{S}\left(V_{s} \mathrm{~W}\right)=\left(\frac{\mathrm{r}}{\mathrm{n}-1}+1\right) \mathrm{g}(\mathrm{V}, \mathrm{W})-\left(\frac{\mathrm{r}}{\mathrm{n}-1}+\mathrm{n}\right) \eta(\mathrm{V}) \mathrm{\eta}(\mathrm{W}) .
$$


Using (2.3), (2.4) and (2.12) the relation (2.11) reduces to

$$
\text { 'C }(\mathrm{U}, \mathrm{V}, \mathrm{W}, \mathrm{Y})=0 \text {. }
$$

From (2.13) it follows that

$$
\mathrm{C}(\mathrm{U}, \mathrm{V}) \mathrm{W}=0 .
$$

Thus we can state the following theorem:

Theorem 1: A P-Kenmotsu manifold $\left(M_{n}, g\right)(n>3)$ satisfying the relation $R(X, Y) . C=0$ is conformally flat and hence is an SP-Kenmotsu manifold. For a conformally symmetric Riemannian manifold, we have $\nabla \mathrm{C}=0[6]$ and hence for such a manifold $\mathrm{R}(\mathrm{X}, \mathrm{Y}) . \mathrm{C}=0$ holds.

Thus we have the following corollary of the above theorem:

Corollary 1: A conformally symmetric P-Kenmotsu manifold $\left(M_{n}, g\right)(n>3)$ is an SP-Kenmotsu manifold.

\section{Acknowledgements}

The authors acknowledge Prof. Kalpana, Banaras Hindu University and Dr. B. Satyanarayana of Nagarjuna University for their consistent academic support in preparing the manuscript.

\section{References}

1. I. Sato, On a structure similar to the almost contact structure, Tensor (N.S.), 30 (1976), 219-224.

2. T. Adati and K. Matsumoto, On conformally recurrent and conformally symmetric P-Sasakian manifolds. TRU Math., 13(1977), 25-32.

3. K. Kenmotsu, A class of almost contact Riemannian manifolds. Tohoku Math. Journal, 24 (1972), 93-103.

4. B. B. Sinha and K. L. Sai Prasad, A class of almost para-contact metric Manifold. Bulletin of the Calcutta Mathematical Society, 87 (1995), 307-312.

5. R. L. Bishop and S. I. Goldberg, On conformally flat spaces with commuting curvature and Ricci transformations. Canad. J. Math., 14(5) (1972), 799-804.

6. M. C. Chaki and B. Gupta, On conformally symmetric spaces, Indian J. of Math., 5(1963), 113-122. 
7. U. C. De and N. Guha, On a type of P-Sasakian manifold. Istambul Univ. Fen Fak. Mat. Der., 51(1992), 35-39.

8. K. L. Sai Prasad, Certain classes of almost contact Riemannian Manifolds, International Mathematical Forum, 16(4) (2009), 773-778.

Received: August 11, 2013 